\title{
An adaptable pentaloop defines a robust neomycin-B RNA aptamer with conditional ligand-bound structures
}

\author{
MUSLUM ILGU, ${ }^{1,2}$ D. BRUCE FULTON, ${ }^{2}$ RAGOTHAMAN M. YENNAMALLI, ${ }^{3,5}$ MONICA H. LAMM, ${ }^{4}$ \\ TANER Z. SEN, ${ }^{3}$ and MARIT NILSEN-HAMILTON ${ }^{1,2,3,6}$ \\ ${ }^{1}$ Ames Laboratory, United States Department of Energy, Ames, lowa 50011, USA \\ ${ }^{2}$ Roy J. Carver Department of Biochemistry, Biophysics and Molecular Biology, ${ }^{3}$ Department of Genetics, Development and Cell Biology, and \\ ${ }^{4}$ Department of Chemical and Biological Engineering, lowa State University, Ames, lowa 50011, USA
}

\begin{abstract}
Aptamers can be highly specific for their targets, which implies precise molecular recognition between aptamer and target. However, as small polymers, their structures are more subject to environmental conditions than the more constrained longer RNAs such as those that constitute the ribosome. To understand the balance between structural and environmental factors in establishing ligand specificity of aptamers, we examined the RNA aptamer (NEO1A) previously reported as specific for neomycin-B. We show that NEO1A can recognize other aminoglycosides with similar affinities as for neomycin-B and its aminoglycoside specificity is strongly influenced by ionic strength and buffer composition. NMR and 2-aminopurine (2AP) fluorescence studies of the aptamer identified a flexible pentaloop and a stable binding pocket. Consistent with a wellstructured binding pocket, docking analysis results correlated with experimental measures of the binding energy for most ligands. Steady state fluorescence studies of 2AP-substituted aptamers confirmed that A16 moves to a more solvent accessible position upon ligand binding while A14 moves to a less solvent accessible position, which is most likely a base stack. Analysis of binding affinities of NEO1A sequence variants showed that the base in position 16 interacts differently with each ligand and the interaction is a function of the buffer constituents. Our results show that the pentaloop provides NEO1A with the ability to adapt to external influences on its structure, with the critical base at position $\mathbf{1 6}$ adjusting to incorporate each ligand into a stable pocket by hydrophobic interactions and/or hydrogen bonds depending on the ligand and the ionic environment.
\end{abstract}

Keywords: aminoglycoside; aptamer; structure

\section{INTRODUCTION}

Nucleic acids, especially RNAs, have diverse functions in living organisms that frequently involve conformational rearrangements during ligand binding and signaling, cotranscriptional folding, catalysis in ribozymes, and the assembly of ribonucleoproteins (Al-Hashimi and Walter 2008; Nick Taylor et al. 2008; Forster et al. 2012). Molecules that interfere with the activity of any of these RNAs are potential drugs, particularly if they can distinguish between eukaryotic and prokaryotic RNAs. Aminoglycosides are an example, being an important class of broad-spectrum antibiotics that target the A-site and interfere with protein synthesis. The aminoglycoside target site is formed by helix- 44 of the small subunit of prokaryotic ribosomes where mRNA and tRNA come in close proximity for amino acid transfer to a growing polypep-

\footnotetext{
${ }^{5}$ Present address: Department of Biochemistry and Cell Biology, Rice University, Houston, TX 77005, USA

${ }^{6}$ Corresponding author

E-mail marit@iastate.edu

Article published online ahead of print. Article and publication date are at http://www.rnajournal.org/cgi/doi/10.1261/rna.041145.113.
}

tide chain (Dahlberg 1989). As a result of aminoglycoside binding, the A-site is distorted and the genetic code is misread, leading to translational cessation and bacterial cell death (Moazed and Noller 1987). However, aminoglycosides also interact with a variety of other natural RNAs including the Rev-responsive element (RRE) (Zapp et al. 1993; Wang et al. 1997) and trans-activating region (TAR) of HIV-1 (Wang et al. 1998), the hepatitis delta virus (HDV) ribozyme (Rogers et al. 1996; Chia et al. 1997), hammerhead (HH) ribozyme (Stage et al. 1995; Tor et al. 1998), RNase P (Mikkelsen et al. 1999), iron response element (IRE) (Lee et al. 2004), site 1 mRNA of thymidylate synthase (TS) (Tok et al. 1999), group 1 self-splicing introns (von Ahsen et al. 1991, 1992), and tRNA $^{\text {Phe }}$ (Mikkelsen et al. 2001). Some of these interactions result in toxicity to mammalian cells and so limit the usefulness of these otherwise very effective antibiotics.

\footnotetext{
(C) 2014 Ilgu et al. This article is distributed exclusively by the RNA Society for the first 12 months after the full-issue publication date (see http:/l rnajournal.cshlp.org/site/misc/terms.xhtml). After 12 months, it is available under a Creative Commons License (Attribution-NonCommercial 4.0 International), as described at http://creativecommons.org/licenses/by-nc/4.0/.
} 
To better understand the interaction between RNA and aminoglycosides, RNA aptamers were selected in vitro that recognize neomycin-B (Wallis et al. 1995), tobramycin (Wang et al. 1996; Werstuck and Green 1998), kanamycinA (Lato et al. 1995; Werstuck and Green 1998), and kanamycin-B (Kwon et al. 2001). These aptamers share similar secondary structural motifs with naturally occurring RNAs without sequence similarity. In some cases, aminoglycosidebinding RNA aptamers display higher affinities for aminoglycosides other than the targets against which they were selected (Lato et al. 1995; Wang and Rando 1995; Kwon et al. 2001).

RNA aptamers have great potential in applications inside the cell, especially as riboswitches for synthetic biology. To properly design and optimize synthetic riboswitches, it is extremely valuable to understand how the aptamer interacts with its ligand(s) in the environment in which it will be applied. Neomycin-B binding aptamers have been successfully selected in yeast and applied as riboswitches (Weigand et al. 2011), but little is known about the structures they adopt intracellularly and how they interact with their ligands under these conditions.

The focus of the current study is a 23-nt RNA aptamer selected against neomycin-B (NEO1A), which is the smallest RNA motif reported to have high affinity and specificity for neomycin-B over paromomycin (Wallis et al. 1995; Wallis and Schroeder 1997; Cowan et al. 2000; de-los-SantosAlvarez et al. 2007, 2009; Stampfl et al. 2007). An NMR-derived structure is available for the NEO1A in complex with neomycin-B (Jiang et al. 1999) but there is no equivalent structure for the apo-aptamer. To better understand the interaction of neomycin-B with NEO1A as it would occur inside cells where the aptamer might be applied in a regulatory role, we examined the aptamer-ligand interactions by a variety of means, including affinity measurements (isothermal titration calorimetry [ITC], and 2-aminopurine [2AP] steady state fluorescence), structural analysis $\left(1 \mathrm{D}{ }^{1} \mathrm{H}\right.$ and $2 \mathrm{D}$ NMR, 2AP fluorescence yield, analysis of sequence variants), and computational analysis by molecular docking. Our results show that the interaction of NEO1A with aminoglycosides involves a coordinated interaction between aptamer and ligand in which a mutable pentaloop plays an important role in determining ligand specificity as influenced by the ionic environment and identity of the ligand. In addition, for the first time we report that the specificity of this aptamer under salt conditions that approximate those in a mammalian cell is broader than previously reported and includes high-affinity binding for paromomycin and ribostamycin in addition to neomycin-B. The interaction of aptamer with aminoglycoside ligand appears to involve a "lock and key" mode with a preformed pocket and an "induced fit" interaction with the loop. The nature of the latter interaction is shown here to be a function of the ionic environment and is proposed to involve a switch between hydrogen bonding (H-bonds) and hydrophobic interactions between the loop and the ligand.

\section{RESULTS}

\section{Influence of buffer composition on the neomycin-B aptamer (NEO1A) affinity for the neomycin class of aminoglycosides with ionic strength a prominent factor}

With the goal of applying RNA aptamers in an intracellular environment, we determined the affinity of the NEO1A to aminoglycosides by ITC using a buffer with ionic conditions that resemble those inside the mammalian cell (Buffer A, Supplemental Table S1). When tested previously in a high ionic strength cacodylate-based buffer (Buffer F, Supplemental Table S1), NEO1A was reported to bind neomycin-B with $>100$-fold higher affinity than it binds to paromomycin (Wallis et al. 1995; Wallis and Schroeder 1997). A comparison of NEO1A-binding parameters for its ligands in Buffers $\mathrm{A}$ and $\mathrm{F}$ confirmed the specificity for neomycin-B in Buffer F (Table 1; Wallis et al. 1995) but showed similar affinities for the three neomycin-class aminoglycoside members in Buffer A (Table 1; Supplemental Fig. S1). Thus, interactions between NEO1A and aminoglycosides are influenced by the ionic surroundings.

To evaluate the impact of the ionic strength [I] on the NEO1A interaction with its ligands, we determined its relation with the affinity of NEO1A for aminoglycosides in a buffer series derived from Buffer F (Supplemental Table S2). The relation between $\log \left(K_{\mathrm{a}}\right)$ and $\log [\mathrm{I}]$ is linear for paromomycin and ribostamycin but appears to be nonlinear for neomycin$\mathrm{B}$ (Fig. 1). Applying polyelectrolyte theory by the following linear relationship: $\log K_{\mathrm{a}}=\log K^{0}-m \psi \log [\mathrm{I}]$, where $K_{\mathrm{a}}$ is the measured association constant, $K^{0}$ is the limiting equilibrium constant, $m$ is the number of ion pairs formed between drug and host RNA, and $\psi$ is the thermodynamic counter-ion binding parameter for the host RNA (Stampfl et al. 2007),

TABLE 1. Comparison of the thermodynamic parameters for NEO1A binding to aminoglycosides in two buffers

\begin{tabular}{|c|c|c|c|c|c|}
\hline Aminoglycoside & Buffer & $K_{\mathrm{d}}(\mu \mathrm{M})$ & $\begin{array}{c}\Delta \mathrm{G} \\
(\mathrm{kcal} / \\
\mathrm{mol})\end{array}$ & $\begin{array}{c}\Delta \mathrm{H} \\
(\mathrm{kcal} / \\
\mathrm{mol})\end{array}$ & $\begin{array}{c}\mathrm{T} \Delta \mathrm{S} \\
(\mathrm{kcal} / \\
\mathrm{mol})\end{array}$ \\
\hline \multirow[t]{3}{*}{ Neomycin-B } & A & $0.29 \pm 0.054$ & -9.0 & -14.6 & -5.6 \\
\hline & $\mathrm{F}$ & $0.18 \pm 0.048$ & -9.2 & -16.2 & -7.0 \\
\hline & F/A & 0.62 & & & \\
\hline \multirow[t]{3}{*}{ Paromomycin } & A & $1.4 \pm 0.18$ & -8.0 & -11.6 & -3.6 \\
\hline & $\mathrm{F}$ & $10 \pm 3.0$ & -6.8 & -7.2 & -0.4 \\
\hline & F/A & 7.4 & & & \\
\hline \multirow[t]{3}{*}{ Ribostamycin } & A & $0.48 \pm 0.075$ & -8.6 & -10.6 & -2.0 \\
\hline & $\mathrm{F}$ & $2.2 \pm 0.10$ & -7.7 & -8.6 & -0.9 \\
\hline & $F / A$ & 4.5 & & & \\
\hline
\end{tabular}

The dissociation constants $\left(K_{\mathrm{d}}\right)$ and thermodynamic parameters are shown for neomycin-B, paromomycin, and ribostamycin binding to NEO1A in Buffers $\mathrm{A}$ and $\mathrm{F}$. The $K_{\mathrm{d}} \mathrm{S}$ for neomycin-B and paromomycin in Buffer A were previously reported (llgu et al. 2013). $\mathrm{F} / \mathrm{A}$ is the $K_{\mathrm{d}}$ in Buffer $\mathrm{F}$ divided by the $K_{\mathrm{d}}$ in Buffer $\mathrm{A}$ for each aminoglycoside. 

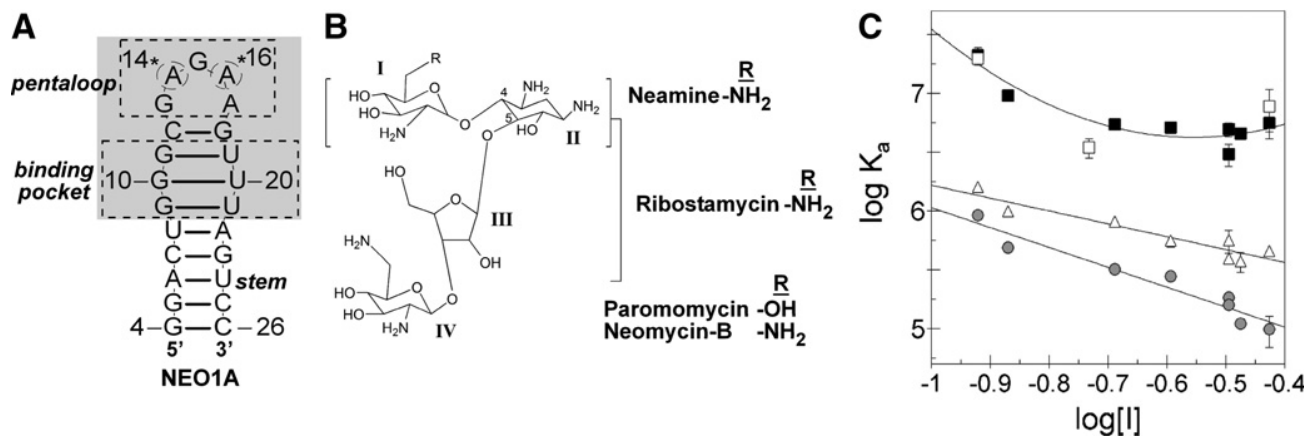

FIGURE 1. The effect of [I] on the interaction between neomycin-class aminoglycosides and NEO1A. $(A)$ The secondary structure of NEO1A was predicted using RNAstructure4.6 (Mathews et al. 2004). For consistency with the previous NMR study, we adopted the numbering used in Jiang et al. (1999) in which the first base is labeled as 4 . The dashed lines denote the previously identified binding pocket and pentaloop. The gray box defines the aminoglycoside binding pocket identified in this study. The adenines at the 14th and 16th positions are circled that were replaced in the NEO1A variants used for 2AP fluorescence measurements. $(B)$ Chemical structures of the neomycin-class aminoglycosides. The neomycin-class of aminoglycoside antibiotics have a 2-deoxystreptamine (2-DOS, ring II), disubstituted at positions 4 and 5, and the substitutions at the R position are indicated for neomycin-class aminoglycosides. (C) A plot of $\log \left(K_{\mathrm{a}}\right)$ vs. $\log (\mathrm{I})$ of NEO1A for neomycin-B (filled squares), ribostamycin (open triangles), and paromomycin (gray circles) obtained in Buffer F at different [I] and neomycin-B (open squares) in Buffer A at different [I]. Refer to Supplemental Table S2 for the buffers used to establish the effect of [I].

resulted in the prediction of up to two ionic contacts involved in the binding of ribostamycin by NEO1A and up to three ionic contacts in the paromomycin-NEO1A interaction. For neomycin- $\mathrm{B}$ binding the nonlinear relation suggests that the aptamer shifts in its interaction mode with [I] of the buffer.

NMR shows a well-structured unoccupied apo-aptamer with clear conformational rearrangement of the bases located in the pentaloop upon aminoglycoside binding

To identify structural elements of the aptamer that reorganize upon ligand binding, we examined $1 \mathrm{D}{ }^{1} \mathrm{H}$, TOCSY, and
NOESY spectra of apo-NEO1A and NEO1A in complex with each of three aminoglycoside ligands. The NMR spectra of the neomycin-B-NEO1A complex matched well with the published spectra for the same complex (Jiang et al. 1999), although some peaks were less intense and broader in our spectra (Fig. 2). These less well-defined spectra are probably due to the difference in temperature of the previous (278K) and our (298K) NMR measurements. Nonetheless, the experimental and published spectra were similar enough to facilitate spectral assignments and to strongly indicate that the neomycin-B-NEO1A complex adopted the published structure (Jiang et al. 1999).
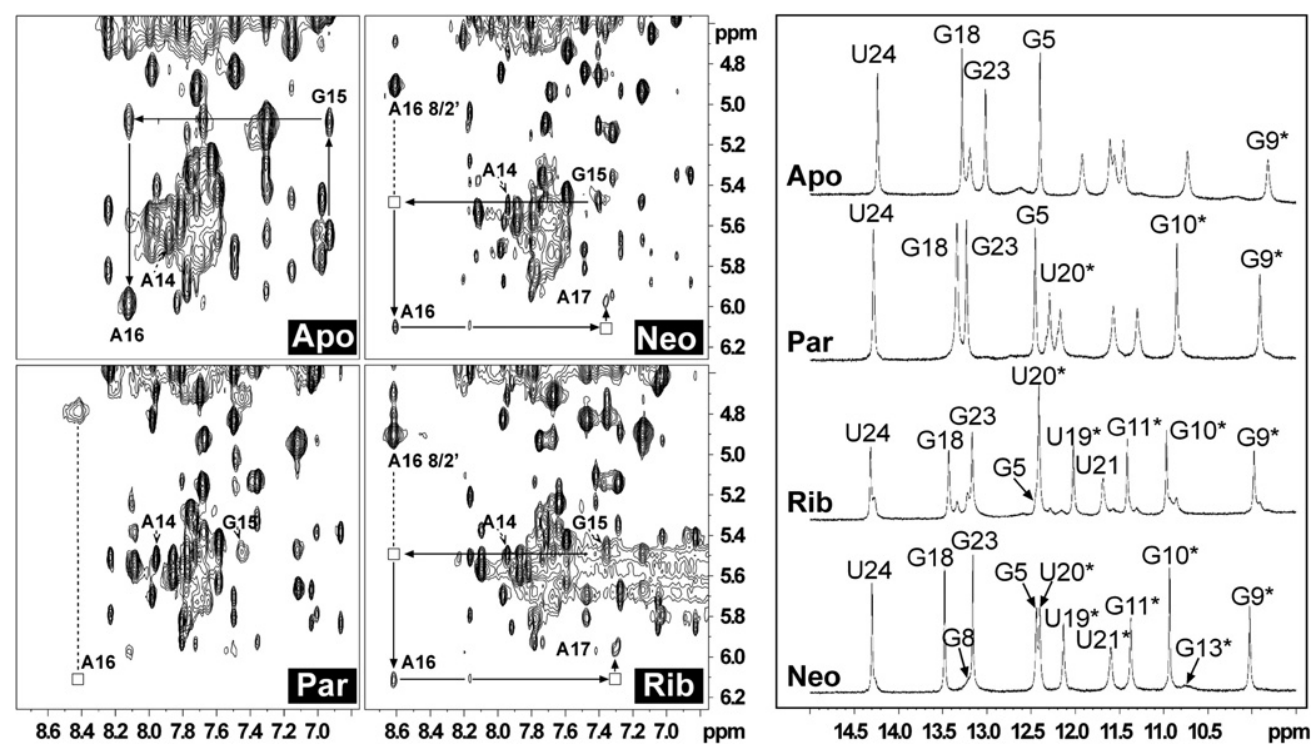

FIGURE 2. NMR spectra of NEO1A alone or in complex with ligands. (Left) 2D NMR NOESY. (Right) Imino protons involved in Watson-Crick base pairs and the G-U wobble pairs. (Apo) no ligand; (Neo) neomycin-B; (Par) paromomycin; (Rib) ribostamycin. 
The imino region spectra for apo-NEO1A and the aminoglycoside complexes are remarkably similar, suggesting the presence of the same $\mathrm{H}$-bond architecture of base-pairings in all complexes and in apo-NEO1A (Fig. 2, right). The imino hydrogen region of the $1 \mathrm{D}{ }^{1} \mathrm{H}$ spectra for neomycin-BNEO1A is nearly identical to the published spectrum but with a weaker line for G13 at $10.75 \mathrm{ppm}$. Twelve imino resonances could be observed and assigned, consistent with four Watson-Crick base pairs in the stem region (G5-C25, A6U24, C7-G23, U8-A22), and in the ligand-binding pocket region three G-U pairs (G9-U21, G10-U20, G11-U19), a Watson-Crick pair (C12-G18) and a noncanonical pair G13-A17. No imino resonance was observed for the terminal base G4. The broadening of three pocket region resonances for the paromomycin-NEO1A complex and all pocket region resonances for apo-NEO1A, suggests that the aminoglycoside binding pocket region is more loosely structured in these two cases.

Comparison of the apo-NEO1A NOESY spectrum with the corresponding spectra for the ligand-aptamer complexes showed all four structures to be virtually identical in the stem-bulge region. The main differences among these structures are in the pentaloop region of NEO1A. This region is essentially identical in NEO1A when complexed with either neomycin-B or ribostamycin. In comparison, the pentaloop in the NEO1A-paromomycin complex was reproducibly less well structured (Fig. 2; Supplemental Fig. S2). In all three complexes the A16 H8 resonances were broadened at unusual downfield chemical shifts and lacked NOEs to $\mathrm{H1}^{\prime}$ on the $5^{\prime}$ sequential sugar. These are all hallmarks of the flipped A16 conformation as observed in the solved NEO1A-neomycin-B structure (Jiang et al. 1999). In contrast, A16 H8 resonances for apo-NEO1A are relatively sharp, at a normal chemical shift and show normal $\mathrm{H}^{\prime}$ NOE connectivities, indicating that A16 is not in a flipped conformation in apoNEO1A. There were also differences among the NMR data for the three bound forms. First, the chemical shifts for most resonances were remarkably similar for the neomycin-B and ribostamycin complexes, whereas there was less chemical-shift similarity for the paromomycin complex. Second, NOEs were observed between A16 and the ligand for the neomycin-B and ribostamycin complexes, but none were observed for the paromomycin complex.

\section{Molecular docking predicts broad aminoglycoside specificity for NEO1A}

The observation by NMR that the binding pocket of NEO1A adopts essentially the same structure in the apo-aptamer compared with the aptamer-ligand complexes suggested a preformed pocket that should be amenable to computational analysis by docking approaches in which the RNA is treated as a rigid body. We used the software DOCK, which was modified for a nucleic acid-ligand system with a set of available RNA-small molecule complexes previously solved by X- ray crystallography (Lang et al. 2009). Ten aminoglycosides were analyzed using DOCK. The estimated docking scores, which are directly proportional to the relative predicted binding strength, were plotted against the $\Delta \mathrm{G}^{\circ}$ s of binding obtained experimentally by ITC (Fig. 3A; Supplemental Fig. S3; Supplemental Tables S3, S4). For nine of the 10 aminoglycosides there was a good correlation between the docking score and measured $\Delta G^{\circ}$ of binding. However, the data for ribostamycin deviated from the derived linear fit (Fig. 3A, gray triangle) and statistical analysis showed it to be an outlier (Supplemental Material). These results suggest that an invariant binding pocket structure is the major contributor to stabilizing the interaction between NEO1A and most aminoglycosides but additional structural feature(s) of NEO1A that
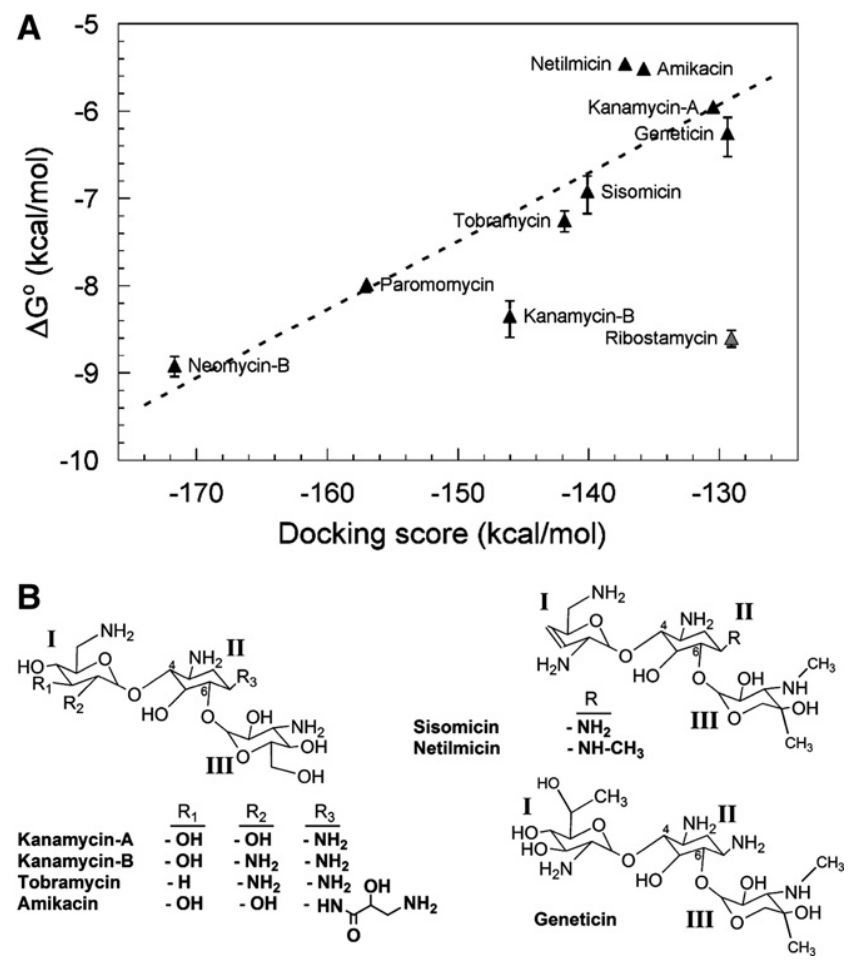

FIGURE 3. Correlation of the predicted docking scores with experimentally obtained binding free energies $\left(\Delta \mathrm{G}^{\circ}\right)$ of aminoglycosides interaction with NEO1A. (A) The free energies of interaction $(\mathrm{kcal} / \mathrm{mol})$ of NEO1A with the aminoglycosides were determined in Buffer $\mathrm{A}$ by ITC and plotted against the docking scores predicted for NEO1A binding to the same aminoglycosides. A statistical analysis of the values identified ribostamycin (gray triangle) as an outlier (Supplemental Material) and it was excluded from the regression fit. The $R^{2}$ for fitting the remaining points was 0.72. (B) Chemical structures of the kanamycinclass aminoglycosides and three others derived from them. The kanamycin-class aminoglycosides have substitutions at positions 4 and 6 with the main substitutions $\mathrm{R} 1$ and $\mathrm{R} 2$ positions on the ring $\mathrm{I}$ as shown. Sisomicin and netilmicin differ with only a single substitution at the $\mathrm{R}$ position on 2-DOS (ring II) as indicated. Geneticin differs from the other aminoglycosides in that it has an extra methyl group attached to C6 on the ring I, and the two other methyl groups attached to ring III, one on the amino group at the third position and the other at the fourth position. 
may vary with ligand-aptamer complex are required for ribostamycin binding.

\section{Spectroscopic analysis of NEO1A variants containing 2AP substitutions in the loop identifies a shift in the environment of these bases upon aminoglycoside binding}

The role of the pentaloop in aptamer ligand selectivity was investigated with NEO1A variants in which A16 or A14 were replaced by 2AP (2AP16NEO1A and 2AP14NEO1A). Binding of neomycin-B, paromomycin, or ribostamycin was accompanied by increased fluorescence of 2AP16NEO1A and decreased fluorescence of $2 \mathrm{AP} 14 \mathrm{NEO} 1 \mathrm{~A}$ with the two sets of changes also showing a qualitatively inverse pattern (Fig. 4A,B). Quantitatively, the change in fluorescence at saturation was greatest for neomycin-B binding and the smallest for paromomycin with both 2AP base substitutions. The absolute change with ribostamycin was similar to neomycin-B for 2AP16NEO1A and to paromomycin for 2AP14NEO1A. These differences between the ligand-NEO1A interactions further support the notion that the structure of each NEO1A-ligand complex is unique.

The relative changes in 2AP16 fluorescence quenching with ligand binding were substantial when compared with the fluorescence of 2AP (free base) but were smaller in both Buffers $\mathrm{A}$ and $\mathrm{F}$ for paromomycin binding compared with the binding of either neomycin-B or ribostamycin (Fig. 4C). The results of the 2AP16 fluorescence yield relative to $2 \mathrm{AP}$ in conjunction with the interpretations of the NMR spectra suggest that 2AP16 in apo-NEO1A is in a stacked configuration. The reciprocal changes in fluorescence yield of 2AP14 and 2AP16 on ligand binding show that, in the RNA-aminoglycoside complex, 2AP16 spends more time and 2AP14 spends less time in a hydrophilic environment. If tetraloop configurations are present in apo-NEO1A as in the NEO1A-ligand complexes, then ligand binding likely re- sults in an exchange of A14 for A16 in the stack, albeit not in precisely the same configuration.

Although 2AP has been previously shown to be a good reporter of nucleic acid structure when substituted for adenosine (Sowers et al. 1986; Nordlund et al. 1989; Wilhelmsson 2010), this base has different H-bonding coordinates compared with adenosine. The solved structure of NEO1A is consistent with a potential H-bond between A16 and the ligand (Jiang et al. 1999), which is supported by our NMR data for the neomycin-B and ribostamycin complexes. If the pentaloop in NEO1A is important for ligand binding and an $\mathrm{H}$ bond contributes to stability of the complex, then 2AP substitutions in the pentaloop might alter the stability of the aptamer-ligand complexes. We found that 2AP16 substitution affects binding affinity differently depending on ligand and buffer (Table 2; Supplemental Tables S5, S6). Whereas substitution of 2AP for A16 did not change the affinity of NEO1A for neomycin-B in Buffer A, it resulted in a 10-fold decrease in affinity for neomycin-B in Buffer $\mathrm{F}$, a decrease in affinity for ribostamycin in both buffers, but a small-tononexistent change in paromomycin binding. The lack of effect of 2AP substitution on the neomycin-B affinity in Buffer $\mathrm{A}$ is not consistent with the existence of an $\mathrm{H}$-bond between the loop base and the ligand under these conditions.

\section{The specificity of aminoglycoside binding to NEO1A is determined by the mode of interaction between the nucleotide base at the 16th position and the ligand}

To further examine the role of A16 in ligand binding, we tested the effects of base substitutions and deletions (Fig. 5; Supplemental Table S5). As predicted from the results of 2AP substitution, the aptamer affinity for neomycin-B was unaffected by the identity of the base in position 16 and the affinity for paromomycin was little affected in Buffer A (Fig. 5). However, the affinity for ribostamycin in Buffer A depended on the base in position 16. The larger effect of
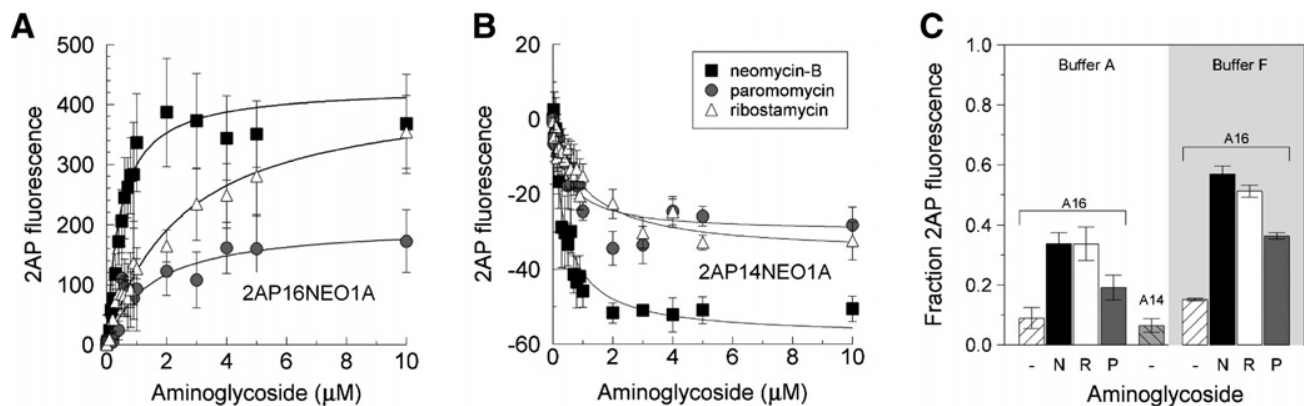

FIGURE 4. $2 \mathrm{AP}$ fluorescence upon aminoglycoside binding to $2 \mathrm{AP}-\mathrm{NEO} 1 \mathrm{~A}$ with substitutions at the A16 or A14 positions. $(A, B) 1 \mu \mathrm{M}$ 2AP16NEO1A $(A)$ or 2AP14NEO1A $(B)$ were titrated with aminoglycosides and fitted for 2AP fluorescence using a hyperbolic model. (C) The fraction 2AP fluorescence obtained from the 2AP16NEO1A (A16) or 2AP14NEO1A (A14) alone (-) or with aminoglycosides at saturating concentrations in Buffer A (white background; $10 \mu \mathrm{M}$ neomycin-B, paromomycin, or ribostamycin) or Buffer $\mathrm{F}$ (gray background; $10 \mu \mathrm{M}$ neomycin-B, $100 \mu \mathrm{M}$ paromomycin or ribostamycin). The aptamer concentrations were $1 \mu \mathrm{M}$ (Buffer A) and $0.5 \mu \mathrm{M}$ (Buffer F). The fluorescence output was normalized to $2 \mathrm{AP}$ fluorescence measured in the respective buffers with 0.5 and $1 \mu \mathrm{M} 2 \mathrm{AP}$. (N) neomycin-B (filled squares); (R) ribostamycin (open triangles); and $(\mathrm{P})$ paromomycin (gray circles). 
TABLE 2. Comparison of dissociation constants for aminoglycoside binding to 2AP16NEO1A and NEO1A in two buffers

\begin{tabular}{lccllll}
\hline & \multicolumn{2}{c}{ Buffer $A$} & & \multicolumn{2}{c}{ Buffer $F$} \\
\cline { 2 - 3 } \cline { 6 - 6 } & 2AP16- & 2AP16- & & 2AP16- & 2AP16- \\
& NEO1A & NEO1A & & NEO1A & NEO1A \\
Aminoglycoside & $K_{\mathrm{d}}(\mu \mathrm{M})$ & NEO1A & & $K_{\mathrm{d}}(\mu \mathrm{M})$ & NEO1A \\
\hline Neomycin-B & $0.27 \pm 0.0063$ & 1.0 & & $1.9 \pm 0.059$ & 10 \\
Paromomycin & $2.8 \pm 0.089$ & 2.0 & & $11 \pm 2.2$ & 1.1 \\
Ribostamycin & $2.6 \pm 0.17$ & 5.3 & & $14 \pm 1.2$ & 6.4 \\
\hline
\end{tabular}

The dissociation constants $\left(K_{\mathrm{d}}\right)$ for neomycin-B, paromomycin and ribostamycin binding to $2 \mathrm{AP} 16 \mathrm{NEO} 1 \mathrm{~A}$ in Buffers $\mathrm{A}$ and $\mathrm{F}$. The ratios are the relative affinities (2AP16NEO1A/NEO1A) for each aminoglycoside in respective buffers. See Table 1 for NEO1A $K_{d} s$.

substitution with $\mathrm{G}$ or $\mathrm{U}$ for A suggests that $\mathrm{H}$-bonding is more important than hydrophobic interactions for stabilizing the ribostamycin-aptamer complex than it is for stabilizing either the neomycin-B or paromomycin-aptamer complexes. Deletion of A16 or A14 decreased aptamer affinity for all ligands (Fig. 5). These results show that the additional base in the pentaloop is essential for ligand interaction, but that the mode of interaction varies with buffer and ligand.

Different results were obtained when the substituted NEO1A variants were tested for their neomycin-B affinity in Buffer F (Fig. 5; Supplemental Table S7). Unlike in Buffer $\mathrm{A}$, the neomycin-B affinity in Buffer $\mathrm{F}$ was decreased by base substitutions, particularly G, U, and 2AP. These results are consistent with the observation of a nonlinear relation between $\log \left[K_{\mathrm{a}}\right]$ and $\log [\mathrm{I}]$ for neomycin-B (Fig. 1) and suggest that the mode of interaction of A16 in the pentaloop is adaptable, either involving $\mathrm{H}$-bonds or hydrophobic interactions depending on the buffer conditions and the ligand.

\section{DISCUSSION}

RNA aptamers that specifically bound to neomycin-B over paromomycin were identified by in vitro selection (Wallis et al. 1995) and this aptamer-ligand interaction has been studied intensively (Wallis and Schroeder 1997; Cowan et al. 2000; de-los-Santos-Alvarez et al. 2007, 2009; Stampfl et al. 2007). With the intent to use NEO1A in synthetic biology applications that would require that the aptamer be functional in the cell cytoplasm, we examined the interaction of NEO1A with its ligands in Buffer A that emulates the free ion concentrations of the mammalian cell cytoplasm. By this means we identified a discrepancy with the previously reported high specificity of NEO1A for neomycin-B over paromomycin. In this study, we show that NEO1A interacts with aminoglycosides by way of a complex that involves coordinated interaction of binding pocket, ligand, and pentaloop, which varies for each ligand and with the ionic environment.

Previous NMR analysis of the NEO1A-neomycin-B structure showed neomycin-B binding as largely determined by the neamine core (rings I and II), which sits deep in a major groove created by three consecutive G-U wobble pairs (Jiang et al. 1999). The complex is stabilized by electrostatic and $\mathrm{H}-$ bonding interactions (Wallis and Schroeder 1997; Cowan et al. 2000; de-los-Santos-Alvarez et al. 2007, 2009; Stampfl et al. 2007). Our NMR results (performed in a similar buffer as the original study) are consistent with the reported structure of the neomycin-B-NEO1A complex. Here we also demonstrate that the structure of the binding pocket is similar in the presence or absence of neomycin-B. Thus, binding of the aminoglycosides to the pocket appears to occur by a "lock and key" mechanism.

The invariance of the pocket observed by NMR is consistent with the observation that aminoglycoside-NEO1A binding affinities, measured in Buffer A, are well correlated with docking scores derived from calculations in which the NMR-derived aptamer structure was treated as a rigid body. Ribostamycin was the one exception for which the measured binding strength deviated significantly from the predicted values. This result may be because, unlike for the other two aminoglycosides in Buffer A, ribostamycin interacts with A16 in the pentaloop by $\mathrm{H}$-bonding, which is not accounted for in the docking algorithm. The determination of similar binding affinities of NEO1A for ribostamycin and neomycin-B in Buffer A is consistent with the previous NMR study showing that ring IV (missing from ribostamycin) plays a minor role in RNA binding (Jiang et al. 1999). Our results for the effects of base replacement on the affinity for aminoglycosides in Buffer A suggest that ribostamycin binding to NEO1A is stabilized by H-bonding with A16, whereas the interactions with neomycin-B and paromomycin are stabilized by hydrophobic

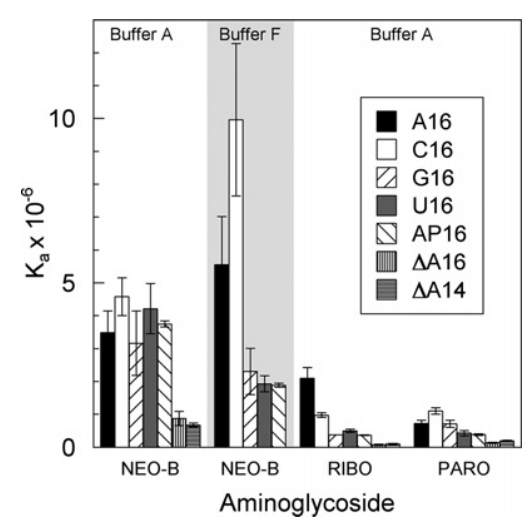

FIGURE 5. Effects of the bases in positions 14 and 16 on aminoglycoside binding and selectivity of NEO1A. Plotted are the affinities of variants of NEO1A that differ by the base in position 16 (adenine: A16, cytosine: C16, guanine: G16, and uracil: U16, or 2-aminopurine: AP16) for aminoglycosides. (NEO-B) neomycin; (RIB) ribostamycin; (PARO) paromomycin. The affinities of NEO1A variants lacking a base in position $16(\triangle \mathrm{A} 16)$ or position $14(\triangle \mathrm{A} 14)$ are also shown. All variants were tested with all ligands in Buffer A. Only A16, C16, G16, U16, and AP16 were tested in Buffer F. The data represent the average association constants $\left(K_{\mathrm{a}}\right)$ obtained from a minimum of two replicate ITC experiments. Refer to the Supplemental Tables S4-S7 for the $K_{\mathrm{d}}$ values for the interaction of NEO1A with each variant. 
interactions (also predicted by Ligplot) that can be provided by any base in this position.

Application of polyelectrolyte theory to the effect of [I] on NEO1A affinities for its ligands suggests that up to two ionic interactions are involved in ribostamycin binding, whereas up to three are involved in paromomycin binding. The relation between $\log \left[K_{\mathrm{a}}\right]$ and $\log [\mathrm{I}]$ was nonlinear for neomycin-B. By comparison, a previous study obtained a linear relation for neomycin-B binding by a 2AP-substituted NEO1A from which 2-3 ionic interactions were predicted (Stampfl et al. 2007). The $5 \mathrm{mM}$ cacodylate ( $\mathrm{pH}$ 6.8) buffer used in this cited study allowed additional lower affinity binding for which we found little evidence in the buffers described here. The conditions used in our studies instead suggest it is likely that the interaction of NEO1A with neomycin-B can alternate between a hydrogen-bond stabilized ligand-loop interaction and one that is independent of $\mathrm{H}$-bonding, presumably mediated by hydrophobic forces.

Our results identify the pentaloop as the means by which NEO1A adjusts to accommodate a range of aminoglycoside ligands and the influence of the ionic environment on ligand binding. The NMR spectra demonstrate that the NEO1A pentaloop undergoes a conformational rearrangement for ligand binding, with large differences observed in chemical shifts of the G15 and A16 resonances between the apoaptamer and aminoglycoside-aptamer complexes. The increase in fluorescence of $2 \mathrm{AP}$ in position 16 also provides evidence for a shift of this base from a hydrophobic (presumably stacked) environment in the apo-aptamer to a hydrophilic environment in the ligand bound states. Thus, as opposed to the "lock and key" interaction with the binding pocket, the ligand appears to interact with the loop by either "induced fit" or "conformational selection."

Substitution of A16 with $2 \mathrm{AP}$ or other bases identified a buffer dependence in the role of this base in ligand interaction. The NMR-derived structure (produced under low [I] conditions in the absence of $\mathrm{Mg}^{2+}$ ) shows A16 lying directly on top of neomycin-B with the possibility of at least one $\mathrm{H}$ bond (Jiang et al. 1999). The results from 2AP16-substituted NEO1A show a significant change in environment of this base with ligand binding that suggests it is less frequently in a stacked position in the aptamer-ligand complex. In Buffer A neomycin-B binding affinity is unaffected by the identity of the base in position 16 , which suggests the absence of H-bonding and that the interaction is stabilized by hydrophobic interactions or that the base does not interact with the ligand under these conditions. In contrast, the affinity for neomycin-B in Buffer F decreased significantly with substitution of $2 \mathrm{AP}, \mathrm{G}$, or $\mathrm{U}$, suggesting that $\mathrm{H}$-bonding is important for neomycin-B binding to NEO1A in Buffer $\mathrm{F}$ but not in Buffer A. This result is the opposite of what is expected at higher [I] if one considers only the interaction of the base at position 16 and the sugar. However, the position of the base over the sugar is a function of the loop, which can form a tetraloop of stacked bases. We propose that the tetra- loop is further stabilized and perhaps more compact at higher [I], which is an expected response to high [I] of a structure that involves many hydrophobic interactions. The disposition of A16 relative to the ligand depends on the loop structure and it is consistent with our results that high [I] results in an alignment of A16 that allows an H-bond between A16 and neomycin-B but not with the other aminoglycosides. At lower [I] the tetraloop stack may relax to an alternate structure that presents A16 in a favorable configuration for hydrophobic bonds with all ligands. The consequence of this proposed balance between hydrophobic or $\mathrm{H}$-bonding interactions between loop and ligand enables the neomycin-Baptamer complex to be relatively invariant to changes in [I]. In contrast, the aptamer interaction with other ligands, which are incapable of H-bond formation with A16, is more sensitive to [I]. The structural flexibility provided by these alternate interactions in the NEO1A-ligand complex results in increased specificity for neomycin-B at high compared with low [I].

Although we cannot rule out the possibility that A16 is freely mobile in a NEO1A-neomycin-B complex in Buffer $\mathrm{A}$, the differences in $\Delta \mathrm{G}$, and consequently $K_{\mathrm{d}}$, are small between Buffers A and F, which suggests the substitution of one mode of stabilization with another. Also, the normalized fluorescence yield of the 2AP16NEO1A is higher in Buffer $\mathrm{F}$ than in Buffer A, which argues for interaction of $2 \mathrm{AP} 16$ with the ligand in both buffers. Overall, our results suggest that the ligand specificity of NEO1A is determined by the interaction between the loop and the ligand, which is mainly mediated by the base in position 16 , and the ionic environment can influence the nature of this interaction.

The decreased sensitivity of neomycin-B affinity to $[\mathrm{I}]$ and the decreased specificity for aminoglycoside structures of the aptamer under low [I] conditions has a significant impact on the applications of this aptamer for synthetic biology applications. First, its lack of sensitivity to [I] for binding neomycin$\mathrm{B}$ allows this aptamer to function at near optimal capacity over a large range of salt concentrations. This is important for the prokaryotic organisms that are generally used for synthetic biology because these organisms experience large changes in intracellular [I], particularly with stress (Brown 1964; Csonka 1989). The resistance to [I] makes this aptamer more reliable under all conditions of growth and organism stress. The second observation of decreased specificity with respect to aminoglycoside is also a large advantage for application of this aptamer because it provides an opportunity to modify the aminoglycoside regulator of the aptamer (perhaps used as a riboswitch) so as to not alter other cellular events. For example, neomycin-B is toxic due to its interaction with the ribosome. But, ribostamycin binds the ribosome very poorly. Thus, our results suggest that ribostamycin would be a better ligand for a neomycin riboswitch. To date, only neomycin- $\mathrm{B}$ has been considered as the aptamer ligand. The recognition that other aminoglycosides also bind this aptamer opens possibilities for developing modified aminoglycosides 
as potential aptamer ligands that could be more appropriate for a particular application. Some molecules can be more readily modified by synthetic routes than others. For the neomycin aptamer, the expansion of available ligands for further modification makes it possible to modify the previously unknown ligand, ribostamycin, for increased cell penetration. Ribostamycin binds the ribosome very poorly and would be more selective for the aptamer over the ribosome than neomycin-B, thereby decreasing the toxicity of a synthetic riboswitch ligand.

It should be noted that, although the aptamer's specificity is relaxed with respect to aminoglycosides in certain environments, such as Buffer A, this is not expected to apply to the interaction of NEO1A with other compounds. This is because the relaxed specificity is due to interaction of a loop with an aminoglycoside sitting in the binding pocket. The binding pocket retains the aminoglycoside specificity, whereas the loop relaxes the selectivity among aminoglycosides. Also, the relevance of our results is not limited to the synthetic biology applications of the neomycin aptamers, but also to other aptamers, particularly as they are being selected and optimized. The process of optimizing aptamers involves many incremental decisions as to which of a number of potential aptamer sequences to choose for further development. Our results suggest that a good choice is to go with structures that are expected to have a loop of five bases, where four can stack in a tetraloop and the fifth base can interact with the ligand or another part of the aptamer to "clamp in" a small ligand. Decision paths that include this consideration are more likely to result in a robust aptamer with more ability to adjust to environmental conditions.

In summary, we show that the neomycin-B aptamer has a preset binding pocket and a flexible pentaloop that adapts to different ligands with alternate interactions that are influenced by its ionic environment. The pentaloop is critical for NEO1A interaction with all ligands. A common feature of aptamers selected to bind aminoglycosides is the presence of a pentaloop with four (GNRA) bases and an extra base that interacts with the aminoglycoside (Wallis et al. 1995). Our results suggest that the mechanism of NEO1A binding to aminoglycosides is a combination of "lock and key" interaction with an invariant pocket that coordinates with an "induced fit" or "conformational selection" interaction with the pentaloop, from which one base folds over the ligand. The flexibility of the loop allows the aptamer to respond to variations in the ionic environment and ligand, thereby providing for a robust aptamer that can recognize neomycin-B in a variety of environments. Thus, the NEO1A-ligand complex has a conditional structure that depends on environmental components and the ligand identity.

In addition to revealing the role of the pentaloop in defining the NEO1A-ligand specificity, this is the first report to our knowledge that this aptamer is not specific for neomycin-B in the presence of salt concentrations similar to those inside mammalian cells where it has applications for synthetic biol- ogy. These results reveal that, in an intracellular environment, NEO1A is likely to bind a variety of aminoglycoside ligands beyond neomycin-B.

\section{MATERIALS AND METHODS}

\section{Chemicals and RNAs}

Neomycin-B, paromomycin, ribostamycin, tobramycin, kanamycin-A, kanamycin-B, sisomicin, geneticin, netilmicin, and amikacin (Figs. 1A, 3B) were obtained as their sulfate salts from SigmaAldrich. All RNA oligonucleotides were from Integrated DNA Technologies Inc., and were maintained at $-20^{\circ} \mathrm{C}$ in deionized distilled water $\left(\mathrm{ddH}_{2} \mathrm{O}\right)$ until use. Sodium cacodylate was from SigmaAldrich and cacodylic acid was from Amresco. All other chemicals were from Fisher Scientific. Buffers were as follows: A $(13.5 \mathrm{mM}$ $\mathrm{NaCl}, 150 \mathrm{mM} \mathrm{KCl}, 20 \mathrm{mM}$ HEPES, $0.22 \mathrm{mM} \mathrm{Na}_{2} \mathrm{HPO}_{4}, 0.44$ $\mathrm{mM} \mathrm{KH}_{2} \mathrm{PO}_{4}, 120 \mu \mathrm{M} \mathrm{MgCl}_{2}, 120 \mathrm{nM} \mathrm{CaCl}, 100 \mu \mathrm{M} \mathrm{MgSO}_{4}$ at $\mathrm{pH}$ 7.3), $\mathrm{N}\left(10 \mathrm{mM} \mathrm{Na}_{2} \mathrm{HPO}_{4}\right.$ at $\mathrm{pH} 7.3$ ), and $\mathrm{F}\left(5 \mathrm{mM} \mathrm{MgCl}_{2}\right.$, $200 \mathrm{mM} \mathrm{NH}_{4} \mathrm{CI}, 80 \mathrm{mM} \mathrm{KCl}, 80 \mathrm{mM} \mathrm{Na}$-cacodylate at $\mathrm{pH} \mathrm{7.4).}$ All buffers were prepared with deionized distilled water and their pH's were measured at $25^{\circ} \mathrm{C}$. Sequences of the RNA aptamers used in this study are shown in Supplemental Table S1. The buffer components used to determine the effect of [I] on aminoglycoside binding are listed in Supplemental Table S2.

\section{Isothermal titration calorimetry (ITC)}

ITC experiments were performed with a Microcal VP-ITC microcalorimeter. Data were analyzed using nonlinear least-squares curve fitting in Origin7.0 (OriginLab Corp.).

\section{Molecular docking of aminoglycosides to the neomycin RNA aptamer}

The fifth model (of nine) of the NMR structure for NEO1A was obtained from the Protein Data Bank as the most representative average structure (PDB ID: 1nem) (Jiang et al. 1999). The Dock Prep module of Chimera v 1.5.2 (Pettersen et al. 2004) was used to prepare the aptamer for docking. Preparation of the ligands for docking involved the addition of hydrogen atoms and assigning partial Gasteiger charges using BABEL v 1.6 (CCL.NET). Molecular docking was performed using DOCK v6.2 (Kuntz et al. 1982). For each ligand, 1000 orientations were used as input and the highly ranked conformation of the ligand along with the DOCK score was obtained as output. Ligplot v4.5.3 (Wallace et al. 1995) was used to map the interactions between the aptamer and the aminoglycosides and to validate the docking predictions.

\section{Measurement of steady state 2-aminopurine (2AP) fluorescence}

The binding of aminoglycosides was monitored by 2AP fluorescence of 2AP16NEO1A and 2AP14NEO1A (in which 2AP was substituted for A16 and A14, respectively) using a Cary Eclipse spectrofluorometer (Varian). Excitation $\left(\lambda_{\mathrm{ex}}\right)$ was $307 \mathrm{~nm}$ and emission $\left(\lambda_{\mathrm{em}}\right)$ was measured at $370 \mathrm{~nm}$. 


\section{Nuclear magnetic resonance (NMR) spectroscopic analysis}

NMR spectra were collected for four samples: the 23-nt NEO1A aptamer alone and the aptamer in the presence of neomycin- $\mathrm{B}$, paromomycin, or ribostamycin. All spectra were acquired at $25^{\circ} \mathrm{C}$. Samples consisted of $500 \mu \mathrm{M}$ NEO1A with $500 \mu \mathrm{M}$ ligand (if present) in $10 \mathrm{mM}$ phosphate buffer ( $\mathrm{pH}$ 7.3) (Buffer N). Samples were dissolved in $95 \% \mathrm{H}_{2} \mathrm{O}, 5 \% \mathrm{D}_{2} \mathrm{O}$ and were dried and redissolved in $100 \% \mathrm{D}_{2} \mathrm{O}$ for some experiments.

$1 \mathrm{D}{ }^{1} \mathrm{H}$ spectra were collected in $95 \% \mathrm{H}_{2} \mathrm{O}, 5 \% \mathrm{D}_{2} \mathrm{O}$ using a 3-9-19 WATERGATE sequence with a shaped flip-back pulse. Free induction decays (FIDs) were sums of 64 scans of $16 \mathrm{~K}$ complex points, processed to yield spectra of $16 \mathrm{~K}$ real points.

2D TOCSY and NOESY spectra were collected in both $95 \% \mathrm{H}_{2} \mathrm{O}$, $5 \% \mathrm{D}_{2} \mathrm{O}$, and pure $\mathrm{D}_{2} \mathrm{O}$. TOCSY spectra were acquired using MLEV-17 isotropic mixing and WATERGATE solvent suppression (Bruker sequence mlevgpph19) with a mixing time of $75 \mathrm{msec}$. NOESY spectra were acquired using a flip-back pulse during mixing and WATERGATE solvent suppression (Bruker sequence noesyfpgpphrs 19) with a mixing time of $200 \mathrm{msec}$. Sixteen and 80 scans were used for the TOCSY and NOESY spectra, respectively. All 2D data were acquired as arrays of $1024 \times 256$ complex points and processed to yield arrays of $1024 \times 512$ real points. NMR data were acquired and processed using Bruker Topspin 2.0 software. The processed spectra were converted to "ucsf" format and analyzed using Sparky 3 software (Goddard and Kneller).

\section{SUPPLEMENTAL MATERIAL}

Supplemental material is available for this article.

\section{ACKNOWLEDGMENTS}

This research was supported by the US Department of Energy, Office of Biological and Environmental Research through the Ames Laboratory. The Ames Laboratory is operated for the US Department of Energy by Iowa State University under Contract No. DE-AC02-07CH11358. We thank Dr. Gulden Camci-Unal and Muneera Beach for advice and technical support in ITC use and data analysis, Dr. Yeon-Jung Seo for assistance with statistical analysis, and Dr. Tianjiao Wang and Lee Bendickson for helpful discussions and technical support.

Received July 5, 2013; accepted February 21, 2014.

\section{REFERENCES}

Al-Hashimi HM, Walter NG. 2008. RNA dynamics: It is about time. Curr Opin Struct Biol 18: 321-329.

Brown AD. 1964. Aspects of bacterial response to the ionic environment. Bacteriol Rev 28: 296-329.

Chia JS, Wu HL, Wang HW, Chen DS, Chen PJ. 1997. Inhibition of hepatitis $\delta$ virus genomic ribozyme self-cleavage by aminoglycosides. J Biomed Sci 4: 208-216.

Cowan JA, Ohyama T, Wang D, Natarajan K. 2000. Recognition of a cognate RNA aptamer by neomycin B: quantitative evaluation of hydrogen bonding and electrostatic interactions. Nucleic Acids Res 28: 2935-2942.
Csonka LN. 1989. Physiological and genetic responses of bacteria to osmotic stress. Microbiol Rev 53: 121-147.

Dahlberg AE. 1989. The functional role of ribosomal RNA in protein synthesis. Cell 57: 525-529.

de-los-Santos-Alvarez N, Lobo-Castanon MJ, Miranda-Ordieres AJ, Tunon-Blanco P. 2007. Modified-RNA aptamer-based sensor for competitive impedimetric assay of neomycin B. J Am Chem Soc 129: $3808-3809$.

de-los-Santos-Alvarez N, Lobo-Castanon MJ, Miranda-Ordieres AJ, Tunon-Blanco P. 2009. SPR sensing of small molecules with modified RNA aptamers: detection of neomycin B. Biosens Bioelectron 24: 2547-2553.

Forster U, Weigand JE, Trojanowski P, Suess B, Wachtveitl J. 2012. Conformational dynamics of the tetracycline-binding aptamer. Nucleic Acids Res 40: 1807-1817.

Goddard T, Kneller D. SPARKY 3. 2008. University of California, San Francisco.

Ilgu M, Wang T, Lamm MH, Nilsen-Hamilton M. 2013. Investigating the malleability of RNA aptamers. Methods 63: 178-187.

Jiang L, Majumdar A, Hu W, Jaishree TJ, Xu W, Patel DJ. 1999. Saccharide-RNA recognition in a complex formed between neomycin B and an RNA aptamer. Structure 7: 817-827.

Kuntz ID, Blaney JM, Oatley SJ, Langridge R, Ferrin TE. 1982. A geometric approach to macromolecule-ligand interactions. J Mol Biol 161: 269-288.

Kwon M, Chun SM, Jeong S, Yu J. 2001. In vitro selection of RNA against kanamycin B. Mol Cells 11: 303-311.

Lang PT, Brozell SR, Mukherjee S, Pettersen EF, Meng EC, Thomas V, Rizzo RC, Case DA, James TL, Kuntz ID. 2009. DOCK 6: combining techniques to model RNA-small molecule complexes. RNA 15: 1219-1230.

Lato SM, Boles AR, Ellington AD. 1995. In vitro selection of RNA lectins: using combinatorial chemistry to interpret ribozyme evolution. Chem Biol 2: 291-303.

Lee J, Kwon M, Lee KH, Jeong S, Hyun S, Shin KJ, Yu J. 2004. An approach to enhance specificity against RNA targets using heteroconjugates of aminoglycosides and chloramphenicol (or linezolid). J Am Chem Soc 126: 1956-1957.

Mathews DH, Disney MD, Childs JL, Schroeder SJ, Zuker M, Turner DH. 2004. Incorporating chemical modification constraints into a dynamic programming algorithm for prediction of RNA secondary structure. Proc Natl Acad Sci 101: 7287-7292.

Mikkelsen NE, Brannvall M, Virtanen A, Kirsebom LA. 1999. Inhibition of RNase P RNA cleavage by aminoglycosides. Proc Natl Acad Sci 96: 6155-6160.

Mikkelsen NE, Johansson K, Virtanen A, Kirsebom LA. 2001. Aminoglycoside binding displaces a divalent metal ion in a tRNAneomycin B complex. Nat Struct Biol 8: 510-514.

Moazed D, Noller HF. 1987. Interaction of antibiotics with functional sites in 16S ribosomal RNA. Nature 327: 389-394.

Nick Taylor J, Darugar Q, Kourentzi K, Willson RC, Landes CF. 2008. Dynamics of an anti-VEGF DNA aptamer: a single-molecule study. Biochem Biophys Res Commun 373: 213-218.

Nordlund TM, Andersson S, Nilsson L, Rigler R, Graslund A, McLaughlin LW. 1989. Structure and dynamics of a fluorescent DNA oligomer containing the EcoRI recognition sequence: fluorescence, molecular dynamics, and NMR studies. Biochemistry 28: 9095-9103.

Pettersen EF, Goddard TD, Huang CC, Couch GS, Greenblatt DM, Meng EC, Ferrin TE. 2004. UCSF Chimera-a visualization system for exploratory research and analysis. J Comput Chem 25: 16051612.

Rogers J, Chang AH, von Ahsen U, Schroeder R, Davies J. 1996. Inhibition of the self-cleavage reaction of the human hepatitis $\delta$ virus ribozyme by antibiotics. J Mol Biol 259: 916-925.

Sowers LC, Fazakerley GV, Eritja R, Kaplan BE, Goodman MF. 1986. Base pairing and mutagenesis: observation of a protonated base pair between 2-aminopurine and cytosine in an oligonucleotide by proton NMR. Proc Natl Acad Sci 83: 5434-5438. 


\section{Ilgu et al.}

Stage TK, Hertel KJ, Uhlenbeck OC. 1995. Inhibition of the hammerhead ribozyme by neomycin. RNA 1: 95-101.

Stampfl S, Lempradl A, Koehler G, Schroeder R. 2007. Monovalent ion dependence of neomycin B binding to an RNA aptamer characterized by spectroscopic methods. Chembiochem 8: 1137-1145.

Tok JB, Cho J, Rando RR. 1999. Aminoglycoside antibiotics are able to specifically bind the $5^{\prime}$-untranslated region of thymidylate synthase messenger RNA. Biochemistry 38: 199-206.

Tor Y, Hermann T, Westhof E. 1998. Deciphering RNA recognition: aminoglycoside binding to the hammerhead ribozyme. Chem Biol 5: R277-R283.

von Ahsen U, Davies J, Schroeder R. 1991. Antibiotic inhibition of group I ribozyme function. Nature 353: 368-370.

von Ahsen U, Davies J, Schroeder R. 1992. Non-competitive inhibition of group I intron RNA self-splicing by aminoglycoside antibiotics. $J$ Mol Biol 226: 935-941.

Wallace AC, Laskowski RA, Thornton JM. 1995. LIGPLOT: a program to generate schematic diagrams of protein-ligand interactions. Protein Eng 8: 127-134.

Wallis MG, Schroeder R. 1997. The binding of antibiotics to RNA. Prog Biophys Mol Biol 67: 141-154.

Wallis MG, von Ahsen U, Schroeder R, Famulok M. 1995. A novel RNA motif for neomycin recognition. Chem Biol 2: 543-552.
Wang Y, Rando RR. 1995. Specific binding of aminoglycoside antibiotics to RNA. Chem Biol 2: 281-290.

Wang Y, Killian J, Hamasaki K, Rando RR. 1996. RNA molecules that specifically and stoichiometrically bind aminoglycoside antibiotics with high affinities. Biochemistry 35: 12338-12346.

Wang Y, Hamasaki K, Rando RR. 1997. Specificity of aminoglycoside binding to RNA constructs derived from the 16S rRNA decoding region and the HIV-RRE activator region. Biochemistry 36: 768-779.

Wang S, Huber PW, Cui M, Czarnik AW, Mei HY. 1998. Binding of neomycin to the TAR element of HIV-1 RNA induces dissociation of Tat protein by an allosteric mechanism. Biochemistry 37: $5549-5557$.

Weigand JE, Schmidtke SR, Will TJ, Duchardt-Ferner E, Hammann C, Wohnert J, Suess B. 2011. Mechanistic insights into an engineered riboswitch: a switching element which confers riboswitch activity. Nucleic Acids Res 39: 3363-3372.

Werstuck G, Green MR. 1998. Controlling gene expression in living cells through small molecule-RNA interactions. Science 282: 296-298.

Wilhelmsson LM. 2010. Fluorescent nucleic acid base analogues. Q Rev Biophys 43: 159-183.

Zapp ML, Stern S, Green MR. 1993. Small molecules that selectively block RNA binding of HIV-1 Rev protein inhibit Rev function and viral production. Cell 74: 969-978. 

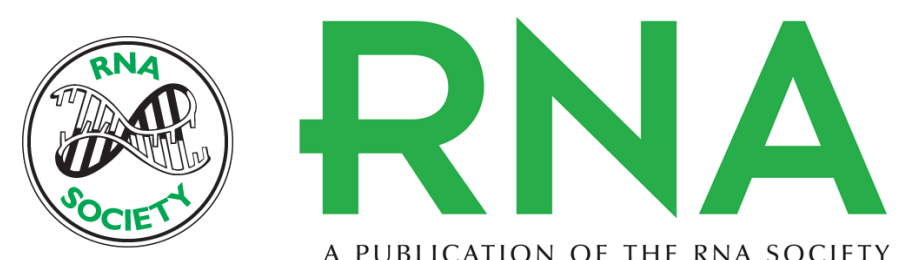

A PUBLICATION OF THE RNA SOCIETY

\section{An adaptable pentaloop defines a robust neomycin-B RNA aptamer with conditional ligand-bound structures}

Muslum Ilgu, D. Bruce Fulton, Ragothaman M. Yennamalli, et al.

RNA 2014 20: 815-824 originally published online April 22, 2014

Access the most recent version at doi:10.1261/rna.041145.113

\section{Supplemental http://rnajournal.cshlp.org/content/suppl/2014/04/03/rna.041145.113.DC1 Material}

References This article cites 42 articles, 8 of which can be accessed free at: http://rnajournal.cshlp.org/content/20/6/815.full.html\#ref-list-1

Creative This article is distributed exclusively by the RNA Society for the first 12 months after the Commons License full-issue publication date (see http://rnajournal.cshlp.org/site/misc/terms.xhtml). After 12 months, it is available under a Creative Commons License (Attribution-NonCommercial 4.0 International), as described at http://creativecommons.org/licenses/by-nc/4.0/.

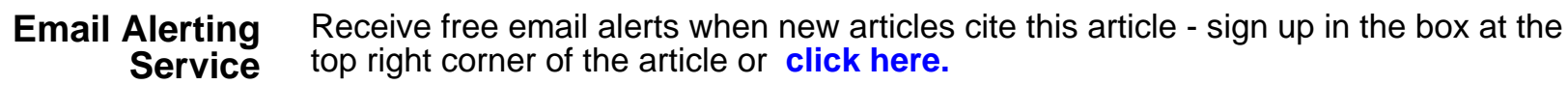

